\title{
Thermodynamics of 4D dS/AdS Gauss-Bonnet black holes from consistent gravity theory in presence of cloud of strings
}

\author{
Hossein Ghaffarnejad 1 \\ Faculty of Physics, Semnan University, P.C. 35131-19111, Semnan, Iran
}

\begin{abstract}
By looking at the Lovelock theorem one can infer that the gravity model given by [18] could not applicable for all type of 4D Einstein Gauss Bonnet (GB) curved spacetimes. Because in 4D space time the Gauss-Bonnet invariant is a total derivative, and hence it does not contribute to the gravitational dynamics. Hence, authors in the work 22 presented an alternative consistent EGB gravity model instead of [18] by applying break of diffeomorphism property. In this work we use it to produce a dS/AdS black hole metric and then investigate its thermodynamic behavior in presence of cloud of Numbo Goto strings.
\end{abstract}

\section{Introduction}

Among the various higher order derivative gravitational models which are given in the literature, Lovelock gravity [1] is quite special because of bing free of ghost [2, 3, 4, 5, 6, 7, 8,. In fact, there are presented many higher order derivative metric theories which show Ostrogradsky instability (see [9, 10] for a good review). In this sense the actions which contains higher order curvature terms introduce equations of motions with fourth order or higher metric derivatives where linear perturbations disclose that the graviton should be a ghost. Fortunately the Lovelock model is free of ghost term and so have field equations involving not more than second order derivatives of the metric. Action functional of the Lovelock gravity, is given by combinations of various terms as follows. The first term is the cosmological constant $\Lambda$, the second term is the Ricci scalar $R=R_{\mu}^{\mu}$, and the third and fourth terms are the second order Gauss-Bonnet [11] and third order Lovelock terms (see Eq. 22 in ref. [12]), respectively. Without the latter term the Lovelock gravity reduces to a simplest form called as the Einstein-Gauss-Bonnet (EGB) theory in which the Einstein-Hilbert action is supplemented with the quadratic

\footnotetext{
${ }^{1}$ E-mail address: hghafarnejad@semnan.ac.ir
} 
curvature GB term as source of self interaction of gravity. Importance of this form of the gravity model is appeared more when we see that it is generated from effective Lagrangian of low energy string theory [13, 14, 15, 16, 17. In fact for higher than 4 dimensions of curved spacetimes the Gauss Bonnet coupling parameter which is calculated by dimensional regularization method have some regular values but not for $D=4$. To resolve this problem the author Glaven and his collaborator presented a proposal [18] but we know now that their initial proposal does not lead to every well-defined gravity theory because regularization is guaranteed just with some of metric theories but not for whole of them. In this sense one can see [19, 20] in which authors explained several inconsistencies of the original paper given by Glavan and Lin [18. Particularly, besides pointing out possible problems in defining the limit or finding an action for the theory, their work also adds new results to the discussion concerning the indefiniteness of second order perturbations even at a Minkowskian background and the geodesic incompleteness of spherically symmetric black hole geometry presented by Glavan and Lin (See also [21]). Thus there must be presented other proposals that can be cover all metric theories. In response to this problem recently a well defined and consistent theory is presented [22] by breaking the diffeomorphism property of the curved spacetime. Instead of the former work [18] the latter model is in concordance with the Lovelock theorem and thereby, seems more to be physical and applicable. For instance FRW cosmology of the latter model is studied in [23] and showed success of this model with respect to the work [18]. In fact there are many papers which are published in the literature about the 4DEGB gravity and its applications in four or higher dimensions of the space time which one can see collections of these works addressed in introduction of the reference [24]. Here we point just to some newest works as follows. For instance one can see [25] where the authors obtained an exact static, spherically symmetric black hole solution in presence of the third-order Lovelock gravity with a string cloud background in seven dimensions when the secondand third-order Lovelock coefficients are related via $\alpha_{2}^{2}=3 \alpha_{3}$. Further, they examined thermodynamic properties of this black hole to obtain exact expressions for mass, temperature, heat capacity and entropy, and also perform the thermodynamic stability analysis. In their work we see that a string cloud background has a profound influence on the horizon structure, thermodynamic properties, and the stability of black holes. Interestingly the entropy of the black hole is unaffected due to the string cloud background. However, the critical solution for thermodynamic stability is affected by the string cloud 
background. Similar work was investigated by Toledo and his collaborator [26] in presence of quintessence but for different spacetime dimensions. They showed the graphs corresponding to the mass and the Hawking temperature for different dimensions of spacetime, $D=4,5,6,7$. By concerning Hawking radiation, it is shown that the radiation spectrum is related to the change of entropy which codifies the presence of the cloud of strings as well as of the quintessence. In their work importance of number of space time dimensions are shown in thermal stabilization of the black holes with effect of string surrounded with quintessence. By studying relation between the Hawking temperature and the entropy they discussed that radiation rate and showed that this quantity depends on the change of entropy which is given in terms of the event horizon and it is strongly influenced by the presence of the cloud of strings as well as of the quintessence. Therefore, the Hawking radiation spectrum depends strongly on the presence of the cloud of strings and on the quintessence. In these view one can infer that presence of string clouds cause to be stable a black hole thermodynamically. Regarding the importance of the role of string theory in the study of black hole dynamics we know that Juan Maldacena (see for a good review [27]), explained for a first time that the development of a string theoretic interpretation of black holes where quantum mechanics and general relativity, theories previously considered incompatible, are united. Work by Maldacena and others has given a precise description of a black hole, which is described holographically in terms of a theory living on the horizon. According to this theory, black holes behave like ordinary quantum mechanical objects-information about them is not lost, as previously thought, but retained on their horizons-leading physicists to look at black holes as laboratories for describing the quantum mechanics of spacetime and for modeling strongly interacting quantum systems. Furthermore the authors of the work [28] used model[18] to obtain EGB spherically symmetric static charged black hole in presence of the Maxwell EM fields and cloud of strings. They obtained that by owing to the corrected black hole due to the background cloud of string, the thermodynamic quantities have also been corrected except for the entropy, which remains unaffected by a cloud of string background. The Bekenstein-Hawking area law turns out to be corrected by a logarithmic area term. The heat capacity diverges to infinity at a critical radius where incidentally the temperature has a maximum, and the Hawking-Page transitions even in absence of the cosmological term is happened by allowing the black hole to become thermodynamically stable. The smaller black holes are globally preferred with negative free energy. Also 
their solution can also be identified as a $4 D$ monopole-charged EGB black hole. Particularly their solution reaches to spherically symmetric black hole solutions of general relativity asymptotically in the limits $\alpha \rightarrow 0$ and absence of the string tension.

In this work we use the consistent EGB gravity model [22] in minisuperspace approach and obtain metric of a spherically symmetric static charge-less black hole in presence of a cosmological parameter and Numbo Goto string tension. Metric field equations are solved numerically in which we use Runge Kutta method to produce numeric values of the fields with best fitting functions. Then we investigated thermodynamic behavior of the obtained solution. To do so we calculate equation of state generated by Hawking temperature of the black hole solution. In fact in the extended phase space the cosmological constant plays an important as thermodynamic pressure of vacuum dS (AdS) Sitter background space. In our obtained metric solutions we will see that the Gauss-Bonnet coupling constant plays a critical role to determine scale of the black hole and positions of the critical points in phase space where the black hole can be participate in the small to large black hole phase transition or Hawking-Page phase transition. In the latter one an unstable black hole reaches to the dS/AdS vacuum space finally.

Setup of this work is as follows. In section 2, we recall consistent 4DEGB gravity given by [22] and use Numbo Goto string fluid as matter source of the system under consideration. In section 3 we generate metric field equations for spherically symmetric 4D black hole line element. In section 4 we solve metric field equations without the string tension where the cosmological constant is just the source. In this case the field equations take on simpler forms and so we obtained analytic form for the metric fields. In order to numerically solve the field equations in presence of the string tension we provide some physical initial conditions in Section 5. In section 6 we apply to numeric analysis of the solutions. Last section denotes to concluding remark and outlook.

\section{$2 \quad 4 \mathrm{D}$ dS/AdS GB gravity with string fluid}

According to the work [22] we know a consistent EGB gravity theory in $D \rightarrow 4$ limit is given by first part of the following action functional.

$$
I=\frac{1}{16 \pi G} \int \mathrm{d} t \mathrm{~d}^{3} x N \sqrt{\gamma}\left(\mathcal{L}_{\mathrm{EGB}}^{4 \mathrm{D}}+\mathcal{L}_{\text {matter }}\right),
$$


in which the Einstein Gauss Bonnet geometrical Lagrangian density is

$$
\begin{gathered}
\mathcal{L}_{\mathrm{EGB}}^{4 D}=2 R-2 \Lambda-\mathcal{M} \\
+\frac{\tilde{\alpha}}{2}\left[8 R^{2}-4 R \mathcal{M}-\mathcal{M}^{2}-\frac{8}{3}\left(8 R_{i j} R^{i j}-4 R_{i j} \mathcal{M}^{i j}-\mathcal{M}_{i j} \mathcal{M}^{i j}\right)\right] .
\end{gathered}
$$

and the second part is matter Lagrangian density. The parameter $G$ is the Newton's gravitational coupling constant, $R=R_{i}^{i}\left(\gamma_{i j}\right)$ and $R_{i j}\left(\gamma_{i j}\right)$ are the Ricci scalar and the Ricci tensor for the spatial metric $\gamma_{i j}$ respectively and

$$
\mathcal{M}_{i j}=R_{i j}+\mathcal{K}_{k}^{k} \mathcal{K}_{i j}-\mathcal{K}_{i k} \mathcal{K}_{j}^{k}, \quad \mathcal{M}=\mathcal{M}_{i}^{i}
$$

with

$$
\mathcal{K}_{i j}=\frac{1}{2 N}\left(\dot{\gamma}_{i j}-2 D_{i} N_{j}-2 D_{j} N_{i}-\gamma_{i j} D_{k} D^{k} \lambda_{\mathrm{GF}}\right) .
$$

Here a dot denotes time derivative $t$ and all the effects of the constraint stemming from the gauge-fixing (GF) are now encoded in Lagrange multiplier $\lambda_{G F} . \quad D_{i}$ is spatial covariant derivative and re-scaled regular GB coupling constant $\tilde{\alpha}$ is defined versus the irregular GB coupling constant $\alpha_{G B}$ in limits of $D \rightarrow 4$ dimensions such that $\tilde{\alpha}=(D-4) \alpha_{G B}$. The above EGB gravity action satisfies the following gauge condition for all spherically symmetric and cosmological backgrounds (see [22] and [23]).

$$
\sqrt{\gamma} D_{k} D^{k}\left(\pi^{i j} \gamma_{i j} / \sqrt{\gamma}\right) \approx 0
$$

In fact the above EGB action is rewritten versus the $1+3 \mathrm{ADM}$ decomposition of the a $4 \mathrm{D}$ background metric for which

$$
d s^{2}=g_{\mu \nu} d x^{\mu} d x^{\nu}=-N^{2} d t^{2}+\gamma_{i j}\left(d x^{i}+N^{i} d t\right)\left(d x^{j}+N^{j} d t\right)
$$

where $N, N_{i}, \gamma_{i j}$ are the lapse function, the shift vector, and the spatial metric respectively. $\gamma$ factor in the action (2.1) is absolute value of determinant of the spatial metric $\gamma_{i j}$. This ADM decomposition is done on the background metric to remove divergent boundary terms of the higher order metric derivative in the GB term of the action functional (2.1) in general 4D form [22]. First term in the theory defined by (2.1) has the time re-parametrization symmetry $t \rightarrow t=t\left(t^{\prime}\right)$. We set matter Lagrangian density $\mathcal{L}_{\text {matter }}$ to be the Nambu-Goto [29] (see also page 100 in ref. [30]) which explains dynamics of relativistic strings as follows.

$$
I_{\mathrm{NG}}=-\int_{\Sigma} \mathcal{L}_{\mathrm{NG}} \mathrm{d} \sigma^{0} \mathrm{~d} \sigma^{1}
$$


in which

$$
\mathcal{L}_{\mathrm{NG}}=\rho \sqrt{\mathfrak{g}}=\rho\left(-\frac{1}{2} \Sigma^{\mu \nu} \Sigma_{\mu \nu}\right)^{\frac{1}{2}}
$$

is lagrangian density and the constant parameter $\rho$ is tension or proper density of the string. Also the bivector $\Sigma^{\mu \nu}$ is related to string worldsheet parameters $\left(\sigma^{0}, \sigma^{1}\right)$ such that

$$
\Sigma^{\mu \nu}=\epsilon^{a b} \frac{\partial x^{\mu}}{\partial \sigma^{a}} \frac{\partial x^{\nu}}{\partial \sigma^{b}}
$$

for which $\epsilon^{a b}$ is two dimensional Levi-Civita tensor density $\epsilon^{01}=-\epsilon^{10}=1$ and $\mathfrak{g}$ is absolute value of determinant of induced metric

$$
\mathfrak{g}_{a b}=g_{\mu \nu} \frac{\partial x^{\mu}}{\partial \sigma^{a}} \frac{\partial x^{\nu}}{\partial \sigma^{b}} .
$$

Stress energy tensor of this relativistic string is given by

$$
T^{\mu \nu}=\mathfrak{g}^{-\frac{1}{2}} \rho \Sigma^{\mu \delta} \Sigma_{\delta}^{\nu}=\frac{2 \partial \mathfrak{L}_{N G}}{\partial g^{\mu \nu}}
$$

and its covariant conservation reads $\nabla_{\mu}\left(\rho \Sigma^{\mu \nu}\right)=0$. To have a $T_{\mu \nu}$ invariant under a re-parametrization of the string's world sheets $\rho$ must be transformed as $\mathfrak{g}^{-1 / 2}$ (see [29] and references therein). Now we are in position to use the above model for a spherically symmetric static black hole spacetime.

\section{4D dS/AdS GBBH surrounded by string cloud}

By comparing the line element (2.6) with general anisotropic form of spherically symmetric static 4D curved metric

$$
d s^{2}=-e^{2 A(r)}\left(1-\frac{2 M(r)}{r}\right) d t^{2}+\frac{d r^{2}}{1-\frac{2 M(r)}{r}}+r^{2} d \theta^{2}+r^{2} \sin ^{2} \theta d \varphi^{2}
$$

we obtain

$$
\begin{gathered}
N=e^{A(r)} \sqrt{1-\frac{2 M(r)}{r}}, \quad N_{r, \theta, \varphi}=0, \\
\gamma_{r r}=\frac{1}{1-\frac{2 M(r)}{r}} \quad \gamma_{\theta \theta}=r^{2}, \quad \gamma_{\varphi \varphi}=r^{2} \sin ^{2} \theta, \quad \lambda_{\mathrm{GF}}=\lambda_{\mathrm{GF}}(r) .
\end{gathered}
$$


By substituting (3.1) into (2.2) we obtain

$\mathcal{L}_{\mathrm{EGB}}^{4 \mathrm{D}}=R\left(\gamma_{i j}\right)-2 \Lambda+12 q^{2}+\frac{\tilde{\alpha}}{2}\left[3 R^{2}(\gamma)+\frac{88}{3} q^{2} R(\gamma)-272 q^{4}-8 R_{i j}(\gamma) R^{i j}(\gamma)\right]$

in which

$$
\begin{gathered}
q(r)=\frac{e^{-A(r)}}{r^{2}}\left[r^{2} \lambda_{G F}^{\prime}(r)\right]^{\prime}, \quad \mathcal{K}_{i j}=-q \gamma_{i j} \\
R(\gamma)=-\frac{4 M^{\prime}}{r^{2}}, \quad R_{i j}(\gamma) R^{i j}(\gamma)=\frac{6}{r^{6}}\left(M-r M^{\prime}\right)^{2}
\end{gathered}
$$

and $/$ denotes derivative with respect to $r$. To obtain explicit form of the lagrangian density of the NG string for the line element (3.1) we should solve $\nabla_{\mu}\left(\rho \Sigma^{\mu \nu}\right)=0$. But this equation is equivalent with $\partial_{\mu}\left(\rho \sqrt{g} \Sigma^{\mu \nu}\right)=0$ because of antisymmetry property of the bivector $\Sigma^{\mu \nu}$. Spherically symmetry property of the space time (3.1) causes to be just $r$ dependent the string lagrangian density. Thus we can show that the equation $\partial_{\mu}\left(\rho \sqrt{g} \Sigma^{\mu \nu}\right)=0$ reads to the following solution for the metric equation (3.1).

$$
\Sigma^{r t}(r)=\frac{c e^{-A(r)}}{\rho r^{2}}, \quad \Sigma_{r t}(r)=-\frac{c e^{A(r)}}{\rho r^{2}}
$$

which by substituting into the lagrangian density (2.8) we obtain

$$
\mathcal{L}_{N G}=\frac{c}{r^{2}}
$$

in which $c$ is an integral constant which should be fixed by physical characteristic of the string namely its tension $\rho$. To do so we calculate the integral equation (2.7) by using a static gauge [30] where the commoving time $\sigma^{0}$ in the parameter space $x^{\mu}\left(\sigma^{0}, \sigma^{1}\right)$ of world sheet is equal to the time $t$ in target space as $\sigma^{0}=t=t_{0}=$ constant and we should assume the static string is along to the radial direction $r$ of world sheet $x^{\mu}\left(\sigma^{0}, \sigma^{1}\right)$ such that

$$
r=r\left(\sigma^{0}, \sigma^{1}\right)=F\left(\sigma^{1}\right), \quad t=\sigma^{0}, \quad \theta\left(\sigma^{0}, \sigma^{1}\right)=\varphi\left(\sigma^{0}, \sigma^{1}\right)=0 .
$$

Here we choose an open string which one edge of the worldsheet to be the curve $\sigma^{1}=0$ and the other edge to be the curve $\sigma^{1}=a$ such that $\sigma^{1} \in$ $[0, a]$ for an open string with arbitrary shape $F\left(\sigma^{1}\right)$. Anyway if the central object is a black hole, the string fluid would naturally be attracted/absorbed by it, and the system would be time-dependent. In order for the string 
fluid to be in equilibrium with the black hole, it must satisfied some specific conditions, like, for example, forming a disk, and with the strings moving on marginally stable orbits outside of the event horizon. Even if we assume that the background metric is spherically symmetric but not static and also string tension is time dependent there is not a doubt about stable time independent metric solutions which we considered here because in Ref.[28] the author proved that spherically symmetric static conditions of a curved spacetime causes to be time independent the NG string cloud stress tensor and it is a general solution of the Einstein metric equation. In other words, we have a "Birkhoff theorem" for the cloud of strings and so the metric solution is the general solution for the symmetry under consideration. In this case nonvanishing components of the induced metric (2.10) reads

$$
\begin{gathered}
\mathfrak{g}_{11}=-e^{2 A(r)}\left(1-\frac{2 M(r)}{r}\right), \quad \mathfrak{g}_{22}=\frac{1}{\left(1-\frac{2 M(r)}{r}\right)}\left(\frac{d F\left(\sigma^{1}\right)}{d \sigma^{1}}\right)^{2}, \\
\sqrt{\mathfrak{g}}=\sqrt{|\operatorname{det}(\mathfrak{g})|}=e^{A} \frac{d F\left(\sigma^{1}\right)}{d \sigma^{1}} .
\end{gathered}
$$

By substituting (3.8) and (3.9) into (2.7) we integrate on the worldsheet $\Sigma$ as

$$
I_{\mathrm{NG}}=-\int_{0}^{t} \mathrm{~d} t \int_{0}^{a} \rho e^{A}\left(\frac{d F\left(\sigma^{1}\right)}{d \sigma^{1}}\right) \mathrm{d} \sigma^{1}=-\int_{0}^{t} \mathrm{~d} t \int_{0}^{r(a)} \rho e^{A} \mathrm{~d} r .
$$

We should now obtain changed form of the above equation from the parameter space of worldsheet to the target spacetime (3.1). This is done by replacing $4 \mathrm{D}$ covariant differential volume element for the line element (3.1) given by

$$
\mathrm{d} v^{[4]}=\sqrt{g} \mathrm{~d} x^{4}=4 \pi r^{2} e^{A} \mathrm{~d} r \mathrm{~d} t
$$

with two dimensional parameter differential surface $\mathrm{d} \sigma^{0} \mathrm{~d} \sigma^{1} \equiv e^{A} \mathrm{~d} t \mathrm{~d} r$ in the above equation. In this sense we obtain

$$
I_{\mathrm{NG}}=-\int\left(\frac{\rho}{4 \pi r^{2}}\right) \mathrm{d} v^{[4]}
$$

which by comparing (3.7) we infer that

$$
\mathcal{L}_{\mathrm{NG}}=\frac{\rho}{4 \pi r^{2}}, \quad c=\frac{\rho}{4 \pi}
$$


By substituting (3.3), (3.2) and (3.13) into the total action functional (2.1) and by integrating angular parts on the 2-sphere $0 \leq \theta \leq \pi, 0 \leq \varphi \leq 2 \pi$ we obtain

$$
\begin{gathered}
I=\frac{1}{4 G} \int \mathrm{d} t \int \mathrm{d} r r^{2} e^{A(r)}\left\{-\frac{4 M^{\prime}}{r^{2}}-2 \Lambda+12 q^{2}-\frac{\rho}{4 \pi r^{2}}\right. \\
\left.-\tilde{\alpha}\left[\frac{176 M^{\prime} q^{2}}{3 r^{2}}+136 q^{4}+\frac{24 M^{2}}{r^{6}}-\frac{48 M M^{\prime}}{r^{5}}\right]\right\} .
\end{gathered}
$$

Euler Lagrange equation for $q$ reads

$$
q\left[12-\tilde{\alpha}\left(\frac{176 M^{\prime}}{3 r^{2}}+136 q^{2}\right)\right]=0
$$

which has two different solutions as

$$
q_{1}=0, \quad q_{2}=\frac{ \pm 1}{\sqrt{136}} \sqrt{\frac{12}{\tilde{\alpha}}-\frac{176 M^{\prime}}{3 r^{2}}} .
$$

Substituting $q_{1,2}$ the equation (3.4) and the action functional (3.14) read to the following forms respectively

$$
\begin{gathered}
\lambda_{\mathrm{GF}}^{(1)}(r) \sim \frac{1}{r} \\
\lambda_{\mathrm{GF}}^{(2)}(r)=\int^{r} \frac{\mathrm{d} r^{\prime}}{r^{\prime 2}} \int^{r^{\prime}}\left(r^{\prime \prime}\right)^{2} q_{2}\left(r^{\prime \prime}\right) e^{A\left(r^{\prime \prime}\right)} \mathrm{d} r^{\prime \prime}
\end{gathered}
$$

and

$$
\begin{gathered}
I_{1}=I_{2}=\frac{1}{4 G} \int \mathrm{d} t \int \mathrm{d} r r^{2} e^{A(r)}\left\{-\frac{4 M^{\prime}}{r^{2}}-2 \Lambda-\right. \\
\left.\frac{\rho}{4 \pi r^{2}}+\tilde{\alpha}\left[-\frac{24 M^{2}}{r^{6}}+\frac{48 M M^{\prime}}{r^{5}}\right]\right\} .
\end{gathered}
$$

The Euler Lagrange equations for the function $A(r)$ and the mass distribution function $M(r)$ reduce to the following relations respectively.

$$
\frac{4 M^{\prime}}{r^{2}}=\frac{-\frac{\rho}{4 \pi r^{2}}-2 \Lambda-\frac{24 \tilde{\alpha} M^{2}(r)}{r^{6}}}{1-\frac{12 \tilde{\alpha} M(r)}{r^{3}}}
$$


and

$$
A^{\prime}(r)=\frac{\frac{-24 \tilde{\alpha} M}{r^{4}}}{1-\frac{12 \tilde{\alpha} M(r)}{r^{3}}} .
$$

Now we are in position to solve the above nonlinear differential equations. This is done via numerical approach in this work. To do so we need physical boundary conditions as follows. We know that the mass distribution function $M(r)$ is related to the matter density function $\rho_{M}$ as

$$
M(r)=\int_{0}^{r} 4 \pi r^{2} \rho_{\mathrm{M}}(r) \mathrm{d} r
$$

for which we can write

$$
\rho_{\mathrm{M}}=\frac{M^{\prime}}{4 \pi r^{2}} .
$$

On the other side for outside region of the gravitational compact object (the vacuum zone) with radius $R$ we have

$$
\rho_{\mathrm{M}}(r)=0, \quad r>R
$$

While for any arbitrary form of the density function $\rho_{\mathrm{M}}(r)$ the mass integral equation (3.22) shows the following boundary condition

$$
M(0)=0
$$

To study dynamics of the compact object it is useful we make dimensionless the equation (3.20) as follows.

$$
\dot{m}(y)=\frac{-\frac{\rho}{16 \pi}-\frac{\Lambda b^{2}}{2} y^{2}-\frac{m^{2}}{y^{4}}}{1-\frac{2 m}{y^{3}}}
$$

where dot ${ }^{*}$ is differentiation with respect to $y$ and we defined

$$
m(y)=\frac{M(r)}{b}, \quad y=\frac{r}{b}, \quad b=\sqrt{6 \tilde{\alpha}}
$$

In this case dimensionless form of the mass density (3.23) and the equation (3.21) reduce to the following forms respectively.

$$
D_{\mathrm{M}}=8 \pi b^{2} \rho_{\mathrm{M}}(y)=\frac{\dot{m}}{y^{2}}=\frac{-\frac{\rho}{16 \pi y^{2}}-\frac{\Lambda b^{2}}{2}-\frac{m^{2}}{y^{6}}}{1-\frac{2 m}{y^{3}}}
$$


and

$$
\dot{A}(y)=\frac{-\frac{4 m}{y^{4}}}{1-\frac{2 m}{y^{3}}} .
$$

We end this section of the paper by giving the radius of the gravitational compact object and corresponding Horizons. Position of radius is obtained by solving $\dot{m}=0$ such that

$$
y_{R}=\frac{ \pm 1}{4} \sqrt{\frac{-\rho \pm \sqrt{\rho^{2}-128 \pi^{2} \Lambda b^{2}}}{\pi \Lambda b^{2}}}
$$

and its horizons are obtained by substituting the horizon hypersurface $y=$ $2 m(y)$ into the equation $(\underline{3.26})$ as

$$
y_{\mathrm{H}_{ \pm}}=\frac{ \pm 1}{\sqrt{1+\frac{\rho}{8 \pi} \mp \sqrt{\left(1+\frac{\rho}{8 \pi}\right)^{2}+2 b^{2} \Lambda}}} .
$$

By substituting the definition

$$
\ell=\frac{y_{\mathrm{H}}}{y_{\mathrm{R}}}
$$

into the above relations we can obtain

$$
\frac{\rho}{4 \pi}=\frac{\ell^{4}-2 y_{\mathrm{H}}^{2}+1}{y_{\mathrm{H}}^{2}\left(1-\ell^{2}\right)}, \quad 2 b^{2} \Lambda=\frac{\ell^{2}\left[\ell^{2}-2 y_{\mathrm{H}}^{2}+1\right]}{y_{\mathrm{H}}^{4}\left(\ell^{2}-1\right)}
$$

where we must be choose $0<\ell<1$ for a star solution and $\ell>1$ for a black hole solution. Applying the positivity condition of the string tension $\rho \geq 0$ on the above relations we obtain

$$
\begin{gathered}
\text { Star: } \quad \ell<1, \quad 2 y_{\mathrm{H}}^{2}<1+\frac{1}{\ell^{4}} \\
\text { Black hole : } \quad \ell>1, \quad 2 y_{\mathrm{H}}^{2}>1+\frac{1}{\ell^{4}} .
\end{gathered}
$$

It is easy to see that for $\mathrm{dS}$ sector $\Lambda>0$ the positive sign in the above horizon positions are valid but in the case of $\operatorname{AdS} \Lambda<0$ both of the positive and negative signs may be valid and reduce to two different real values. In the latter case we call smaller horizon to be black hole horizon and the larger one to be the modified cosmological horizon. Without the cosmological parameter 
$\Lambda=0$ the above equation gives us a curved space time with one horizon such that

$$
y_{\mathrm{H}}=\sqrt{\frac{4 \pi}{8 \pi+\rho}}, \quad \Lambda=0, \quad y_{\mathrm{R}}=\frac{y_{\mathrm{H}}}{\sqrt{2 y_{\mathrm{H}}^{2}-1}} .
$$

We now apply to solve the above dynamical equations for different situations as follows.

\section{Solutions with $\rho=0, \Lambda(>,<,=) 0$}

The equation (3.26) has an analytic exact solution in absence of the string tension as

$$
m(y)=\zeta y^{3}, \quad \zeta=\frac{3 \pm \sqrt{9+10 b^{2} \Lambda}}{10}, \quad \rho=0
$$

for which the equation (3.29) reads

$$
A(y)=\left(\frac{4 \zeta}{2 \zeta-1}\right) \ln (y)
$$

with corresponding metric components

$$
g^{r r}(y)=1-2 \zeta y^{2}, \quad g_{\mathrm{tt}}(y)=-y^{\frac{8 \zeta}{2 \zeta-1}}\left(1-2 \zeta y^{2}\right) .
$$

In this case for $2 \zeta>1$ we have one apparent horizon with position $y_{\mathrm{AH}}=\frac{1}{\sqrt{2 \zeta}}$ and two event horizons with positions $y_{\mathrm{EH}}=0$ and $y_{\mathrm{EH}}=y_{\mathrm{AH}}$. For $0<2 \zeta<1$ position of the event horizon $y_{\mathrm{EH}}$ moves to infinity $y_{\mathrm{EH}} \rightarrow \infty$ and (3.33) reads

$$
\ell=\left(\frac{1}{\zeta}-1\right)^{\frac{1}{4}}
$$

This shows that for $\zeta>\frac{1}{2}$ we have a black hole in which $\ell<1$ and so $y_{\mathrm{R}}<y_{\mathrm{H}}$ while for $0<\zeta<\frac{1}{2}$ we have $y_{\mathrm{R}}>y_{\mathrm{H}}$ and so the above metric is not a black hole but may to be a regular star. The definition of $\zeta$ in the relation (4.1) shows that we must be choose

$$
\Lambda \geq \frac{-3}{20 \tilde{\alpha}} .
$$

This can be understood as the unknown cosmological constant in general theory of relativity originates in fact from the GB coupling parameter and so 
can be understandable. By according to the relationship $\tilde{\alpha}=(D-4) \alpha_{\mathrm{GB}}$ and positivity condition of the GB coupling constant $\alpha_{\mathrm{GB}}>0$ we infer that the above relationship corresponds to a $\mathrm{AdS}(\mathrm{dS})$ space when $\tilde{\alpha}>0(<0)$ and so $D>4(<4)$. This means that when we regularize the GB action functional it should be done by limiting from higher space time dimensions to $4 D$ and vice versa for $\mathrm{dS}$ space. For the above analytic metric solution it is easy to show that the Hawking temperature $\bar{T}=b T=-\frac{1}{2 \pi} \frac{\mathrm{d} g_{\mathrm{tt}}(y)}{\mathrm{d} y}{ }_{\mid y_{\mathrm{H}}}$ reads

$$
\bar{T}=2(2 \zeta)^{\frac{1}{2}\left(\frac{1+6 \zeta}{1-2 \zeta}\right)} .
$$

We know that at the extended phase space, the pressure of AdS space is defined by $P=-\frac{\Lambda}{8 \pi}$ for negative cosmological constant $\Lambda<0$ and $P=\frac{\Lambda}{8 \pi}$ for dS sector with $\Lambda>0$. By regarding these definitions the parameter of $\zeta$ given by (4.1) reads

$$
\bar{P}=b^{2} P=\frac{ \pm\left[(10 \zeta-3)^{2}-9\right]}{80 \pi}
$$

where $+(-)$ corresponds to $\mathrm{dS}(\mathrm{AdS})$ sector. For pressureless space $\Lambda=0$ we have $\zeta=\{0,0.6\}$ for which the corresponding temperature will be $T(0)=0$ and $T(0.6)=0.1422$. Metric field solution is flat Minkowski for $\zeta=0$ but not for $\zeta=0.6$. Figure 1 -a shows event and apparent horizons of the space time in the latter case which their positions are crossing points with the horizontal axes. Event horizon is obtained by solving $g_{\mathrm{tt}}(y)=0$ and apparent horizon is obtained with $g^{\mathrm{rr}}(y)=0$ for spherically symmetric state space times. Pressure-temperature phase diagram for the equations (4.6) and (4.7) are plotted in the figure 1-b,1-c,1-d. These diagrams show a dS/AdS phase transition with coexistence state (butterfly tail form in figures) between them at the crossing point in the P-T diagrams.

\section{$5 \quad$ Initial conditions with $\rho>0, \Lambda(>,<,=) 0$}

For the case $\rho \neq 0$ the equation (3.26) has not analytic solution and it should be solved via numerical methods. To do so we apply Runge Kutta method for which we should assume some physical initial conditions for $m(y), \rho$ and $\Lambda$ and domain of $y$. By looking at the equation (3.25) one can infer that a suitable initial condition for the mass parameter is

$$
m(0)=0
$$


while we are still free to choose various values for $\Lambda, \rho$ and regimes of the variable $y$. To determine that what is suitable regimes for these parameters? we call equation of state of the system by calculating the corresponding Hawking temperature as follows. We know that the Hawking temperature of a black hole space time is determined by value of surface gravity on its exterior horizon such that

$$
T=\left.\frac{-1}{4 \pi} \frac{\mathrm{d} g_{\mathrm{tt}}(r)}{\mathrm{d} r}\right|_{r=2 M(r)}=\frac{e^{2 A(r)}}{2 \pi}\left[A^{\prime}(r)\left(1-\frac{2 M(r)}{r}\right)+\frac{M(r)}{r^{2}}-\frac{M^{\prime}(r)}{r}\right]_{\left.\right|_{\mathrm{r}=2 \mathrm{M}(\mathrm{r})}} .
$$

For AdS (dS) background with pressure $P=\frac{-\Lambda}{8 \pi}>0\left(P=\frac{\Lambda}{8 \pi}>0\right)$ we substitute (3.20) and (3.21) and definition (3.27) into the Hawking temperature (5.2) to obtain the 4 DGB black hole equation of state such that

$$
A d S: \quad \bar{T}=\bar{P} \bar{v}-\bar{v} f(\bar{v}), \quad \bar{v}=\frac{v}{b}=\frac{2 y^{3} e^{2 A(y)}}{1-y^{2}}, \quad 0 \leq y \leq 1
$$

and

$$
d S: \quad \bar{T}=\bar{P} \bar{v}+\bar{v} f(\bar{v}), \quad \bar{v}=\frac{v}{b}=\frac{2 y^{3} e^{2 A(y)}}{y^{2}-1}, \quad y \geq 1
$$

where $\bar{v}$ is dimensionless specific volume and

$$
f[\bar{v}(y)]=\frac{1}{4 \pi y^{2}}\left[\frac{1}{2}+\frac{\rho}{16 \pi}-\frac{1}{4 y^{2}}\right] .
$$

By looking at the above equation of state one can infer that at large scales $y \rightarrow \infty$ the function $f(\bar{v})$ vanishes and so the above equation of state reaches to the well known ideal gas equation of state. Now we are in position to obtain critical point in $\mathrm{T}-\mathrm{v}$ extended phase space by calculating equation of the critical points $\left.\frac{\partial T}{\partial v}\right|_{P}=0$ and $\left.\frac{\partial^{2} T}{\partial v^{2}}\right|_{P}=0$ which reduce to the following conditions.

$$
\left.\frac{\partial \bar{T}}{\partial y}\right|_{P}=0,\left.\quad \frac{\partial^{2} \bar{T}}{\partial y^{2}}\right|_{P}=0
$$

The above equations give us a parametric critical point such that for AdS we have

$$
\begin{aligned}
& A d S: \quad \bar{P}_{\mathrm{c}}\left(y_{\mathrm{c}}\right)=\frac{-3\left(y_{\mathrm{c}}^{4}+2 y_{\mathrm{c}}^{2}+5\right)}{16 \pi y_{\mathrm{c}}^{4}\left(y_{\mathrm{c}}^{4}+10 y_{\mathrm{c}}^{2}-35\right)}, \\
& \bar{v}_{\mathrm{c}}=\frac{2 y_{\mathrm{c}}^{3} e^{2 A\left(y_{\mathrm{c}}\right)}}{1-y_{\mathrm{c}}^{2}}, \quad \bar{T}_{\mathrm{c}}=\frac{\left(y_{\mathrm{c}}^{2}-1\right) e^{2 A\left(y_{\mathrm{c}}\right)}}{\pi y_{\mathrm{c}}\left(y_{\mathrm{c}}^{4}+10 y_{\mathrm{c}}^{2}-35\right)}
\end{aligned}
$$


and for dS

$$
\begin{gathered}
d S: \quad \bar{P}_{\mathrm{c}}\left(y_{\mathrm{c}}\right)=\frac{3\left(y_{\mathrm{c}}^{4}+2 y_{\mathrm{c}}^{2}+5\right)}{16 \pi y_{\mathrm{c}}^{4}\left(y_{\mathrm{c}}^{4}+10 y_{\mathrm{c}}^{2}-35\right)} \\
\bar{v}_{\mathrm{c}}=\frac{2 y_{\mathrm{c}}^{3} e^{2 A\left(y_{\mathrm{c}}\right)}}{y_{\mathrm{c}}^{2}-1}, \quad \bar{T}_{\mathrm{c}}=\frac{\left(y_{\mathrm{c}}^{4}-14 y_{\mathrm{c}}^{2}-11\right) e^{2 A\left(y_{\mathrm{c}}\right)}}{4 \pi y_{\mathrm{c}}\left(y_{\mathrm{c}}^{2}-1\right)\left(y_{\mathrm{c}}^{4}+10 y_{\mathrm{c}}^{2}-35\right)}
\end{gathered}
$$

where for both sectors dS and AdS the string tension satisfies to the following relation.

$$
\rho\left(y_{\mathrm{c}}\right)=\frac{-8 \pi\left(y_{\mathrm{c}}^{6}+7 y_{\mathrm{c}}^{4}-29 y_{\mathrm{c}}^{2}+21\right)}{y_{\mathrm{c}}^{2}\left(y_{\mathrm{c}}^{4}+10 y_{\mathrm{c}}^{2}-35\right)},
$$

for arbitrary parameter $y_{\mathrm{c}}$. By looking at the figure 2-a one can obtain that $\rho=0$ at $y_{\mathrm{c}}= \pm 1$ and $y_{\mathrm{c}}= \pm 1.4432$. Furthermore $\rho>0$ for $0<y_{\mathrm{c}}<1$ and $1.4432<y_{c}<1.6448$. These regions are positioned in the AdS and dS regimes respectively. and satisfy positivity condition on the string tension. Thus to set suitable numeric critical points form the above parametric form consisted with dS and AdS regions we choose the following ansatz.

$$
A d S: y_{\mathrm{c}}=\frac{1}{2}, \bar{T}_{\mathrm{c}}=0.015 e^{2 A(0.5)}, \bar{P}_{\mathrm{c}}=0.16, \rho=44.02, \bar{v}_{\mathrm{c}}=0.33 e^{2 A(0.5)}
$$

and

$$
d S: y_{\mathrm{c}}=\frac{3}{2}, \bar{T}_{\mathrm{c}}=0.21 e^{2 A(1.5)}, \bar{P}_{\mathrm{c}}=-0.023, \rho=3.87, \bar{v}_{\mathrm{c}}=5.4 e^{2 A(1.5)}
$$

where numeric values for $A(0.5)$ and $A(1.5)$ can be obtained by solving the equations (3.26) and (3.29). By substituting the above critical points into the equations (3.33) we obtain two class of solutions which one of them is star $\ell<1$ and the other one is a black hole $\ell>1$ as follows.

$$
\begin{gathered}
A d S: \quad\left\{y_{\mathrm{H}} \approx 0.43, \quad y_{\mathrm{R}} \approx 16.60\right\}_{\mathrm{star}}, \quad\left\{y_{\mathrm{H}} \approx 20.78, \quad y_{\mathrm{R}} \approx 16.60\right\}_{\text {black hole }} \\
d S: \quad\left\{y_{\mathrm{H}} \approx 0.66, \quad y_{\mathrm{R}} \approx 13.09\right\}_{\mathrm{star}}, \quad\left\{y_{\mathrm{H}} \approx 35.50, \quad y_{\mathrm{R}} \approx 13.09\right\}_{\text {black hole }}
\end{gathered}
$$

where we omitted negative roots of the equations (3.33). Because solutions in the regions with $y<0$ are positioned in the analytic continuation of $y$ in the complex algebraic analysis. Also we substitute $P_{\mathrm{c}}=\frac{\Lambda}{8 \pi}$ and $P_{\mathrm{c}}=-\frac{\Lambda}{8 \pi}$ into the above critical points to obtain

$$
b^{2} \Lambda_{\mathrm{AdS}}=-0.006, \quad b^{2} \Lambda_{\mathrm{dS}}=-0.0009 .
$$


for the dS and the AdS cases respectively. We should remember that the black hole choice in (5.12) and the star choice in (5.13) are not physical because for them the thermodynamic specific volume takes on negative sign. Thus in the next section we apply to solve (3.26) and (3.29) just for star choice in the AdS and the black hole choice in the dS sector.

\section{$6 \quad$ Numerical analysis}

In the section 3 we obtained that the spatial regimes $0 \leq y<\infty$ are separated into two subregions such that for dS (AdS) sector we should use $y>1(0<$ $y<1$ ) by omitting analytic continuation regions $y<0$. Also in that section we said that $m(0)=0$ is suitable initial condition for mass function when we solve dynamical equations (3.26) and (3.29) as numerically. In the previous section we obtained consistent initial conditions on $\rho$ and $\Lambda$ parameters of the system under consideration. By substituting (5.10), (5.11) and (5.14) the equation (3.26) reduces to the following form for dS and AdS sectors.

$$
\begin{gathered}
d S: \quad \dot{m}=\frac{-0.077+0.0005 y^{2}-\frac{m^{2}}{y^{4}}}{1-\frac{2 m}{y^{3}}}, \quad y>1 \\
A d S: \quad \dot{m}=\frac{-0.88+0.003 y^{2}-\frac{m^{2}}{y^{4}}}{1-\frac{2 m}{y^{3}}}, \quad 0<y<1
\end{gathered}
$$

for which the equation of state become

$$
\begin{array}{ll}
d S: \quad \bar{T}=\bar{v}\left[\bar{P}+\frac{1}{4 \pi y^{2}}\left(0.58-\frac{1}{4 y^{2}}\right)\right], \quad \bar{v}=\frac{2 y^{3} e^{2 A(y)}}{y^{2}-1} \\
A d S: \quad \bar{T}=\bar{v}\left[\bar{P}-\frac{1}{4 \pi y^{2}}\left(1.38-\frac{1}{4 y^{2}}\right)\right], \quad \bar{v}=\frac{2 y^{3} e^{2 A(y)}}{1-y^{2}}
\end{array}
$$

where $A(y)$ is obtained by solving

$$
\dot{A}=\frac{-\frac{4 m}{y^{4}}}{1-\frac{2 m}{y^{3}}} .
$$

Now we are in position to choose valid domains for $y$ to produce numeric solutions of the fields. By looking at the (5.12) and (5.13) we choose

$$
A d S: \quad 0<y<1, \quad y_{H}=0.43, \quad y_{c}=0.5
$$


and

$$
d S: \quad 1<y<40, \quad y_{H}=35.50, \quad y_{c}=1.5, \quad y_{R}=13.09
$$

We use Runge Kutta method via the Maple and the Mathematica softwares. To produce numeric solutions with this method we should have a suitable form for the dynamical equations as $\frac{d s}{d x}=f(x, s)$. In fact the equation (3.26) has this form but (3.29) has not. To resolve this problem we obtain a consistent differential equation instead of (3.29). This is done for $\dot{A}=w(y)$ just by reproducing $m(y)$ from (3.29) and calculating its $\dot{m}$ and by substituting them into the equation (3.26) such that

$$
\begin{gathered}
\dot{w}=\frac{0.31}{y^{3}}-\frac{0.002}{x}-\left(5 y+\frac{0.5}{y}\right) w \\
+y\left(0.23+1.75 y^{2}\right) w^{2}-y^{2}\left(0.04+0.13 y^{2}\right) w^{3} .
\end{gathered}
$$

To generate a suitable differential equation for $g^{\mathrm{rr}}(y)=S(y)$ we substitute $m=(1-S) y / 2$ and its first derivative into the equation (3.26) such that

$$
\frac{d S}{d y}=-\frac{4 \alpha y^{4}+(2 S+4 \beta-2)+(S-1)^{2}}{y\left(y^{2}+S-1\right)}
$$

where

$$
d S: \quad \alpha=-0.077, \quad \beta=0.0005
$$

and

$$
\text { AdS : } \quad \alpha=-0.88, \quad \beta=0.003 .
$$

By holding the equations (3.26), (6.6) and (6.7), the Maple software takes out the best fitting numeric solutions for $m(y), g^{\mathrm{rr}}(y)$ and $\dot{A}(y)$ given in the figures 3-a,c,d for dS and 4-a,c,e for AdS sectors. Several points on the curves generated by the machine are listed in the tables 1 and 2 and we used them to determine numerical values of the fields $A(y)$ and $g_{\mathrm{tt}}(y)$ via Mathematica software. By looking at these diagrams one can see that the metric fields in both cases of dS and AdS have a crossing point with the horizontal axes which means that they are locations of the black hole horizon. One can infer the most important result from the volume pressure phase diagrams at constant temperatures that: by looking at the figure 3 -f we understand that a dS 4DGB black hole participates in a large to small black hole phase transition for temperatures less that the critical one. For $\bar{T} \geq \bar{T}_{c}$ this black hole at maximal pressure is in a disequilibrium state and it reaches to a vacuum 
AdS finally. At all in both cases of dS and AdS spaces a 4DGB black hole surrounded with a cloud of string take on two phases which may to be in a coexistence state when they have small scales but not in the large scales. In about the AdS 4DGB black hole in presence of the string tension the figure 4-f shows that this black hole at $\bar{T}<\bar{T}_{c}$ is unstable thermodynamically at the maximum pressure and it can be participated in the Hawking-Page phase transition where the black hole evaporates to a vacuum dS finally. For the cases $\bar{T} \geq \bar{T}_{c}$ this black hole does not take on a phase transition.

\section{Conclusion}

In this work we choose EGB gravity model [22] which is consistent in 4D curved spacetimes and solved metric equations for spherically symmetric static black hole line element with and without the cosmological constant and the Numbo Goto string tension. In absence of the string tension we obtained analytic solution for the metric fields but with string tension we used Runge Kutta method to obtain numeric solutions of the fields. By studying thermodynamic of these black holes we infer that for small scales they behave as two fluid systems which for temperatures less than the critical one a dS black hole participates in the large to small black hole phase transition while AdS one reaches to the Hawking-Page phase transition. As extension of this work we like to study in our next work possibility of the Joule-Thomson expansion of this system and other thermodynamic behavior of the obtained metric solution.

Table 1. Numerical solutions for dS pressure

\begin{tabular}{|c|c|c|c|c|c|}
\hline$y$ & $m$ & $10^{7} \times \dot{A}$ & $A$ & $g^{r r}$ & $g_{t t}$ \\
\hline 35 & 16.984 & 0 & 35 & 0.0294857 & $-7.41695 \times 10^{28}$ \\
40 & 20.49 & -1.2216 & 34.99999635 & -0.0245 & $6.16278 \times 10^{28}$ \\
45 & 25.07 & -1.5739 & 34.99999704 & -0.114222 & $2.87317 \times 10^{29}$ \\
50 & 30.24 & -1.60278 & 34.99999780 & -0.2096 & $5.27234 \times 10^{29}$ \\
55 & 36.52 & -1.512854 & 34.99999860 & -0.328 & $8.25062 \times 10^{29}$ \\
60 & 45.03 & -1.38500 & 34.99999939 & -0.501 & $1.26023 \times 10^{30}$ \\
\hline
\end{tabular}

Table 2. Numerical solutions with AdS pressure 


\begin{tabular}{|c|c|c|c|c|c|}
\hline$y$ & $m$ & $\dot{A}$ & $A$ & $g^{r r}$ & $g_{t t}$ \\
\hline 0.42 & 0.21 & 0 & 5.78182 & 0. & 0. \\
0.54 & 0.28 & 4.2 & 5.15252 & -0.037037 & 164.706 \\
0.62 & 0.38 & 4.878 & 4.69953 & -0.225806 & 3896.85 \\
0.768 & 0.506 & 5.194 & 4.71439 & -0.317708 & 10315.2 \\
0.88 & 0.68 & 5.52552 & 4.55532 & -0.545455 & 34368.8 \\
0.998 & 0.897 & 5.6506 & 4.49406 & -0.797595 & 64540.4 \\
\hline
\end{tabular}

\section{References}

[1] D. Lovelock,'The Einstein Tensor and Its Generalizations', J. Math. Phys. (N.Y.)12, 498 (1971).

[2] B. Zwiebach, 'Curvature squared terms and string theories', Phys. Lett. B 156, 315 (1985).

[3] B. Zumino, 'Gravity theories in more than four dimensions',Phys. Rept.137, 109 (1986).

[4] N. Deruelle and L. Farina-Busto, 'Lovelock gravitational field equations in cosmology', Phys. Rev. D41, 3696 (1990).

[5] R. G. Cai and N. Ohta, 'Black Holes in Pure Lovelock Gravities', Phys. Rev. D74, 064001 (2006).

[6] D.G. Boulware, S. Deser, 'String-Generated Gravity Models', Phys. Rev. Lett.55, 2656 (1985).

[7] J.T. Wheeler, 'Symmetric solutions to the Gauss-Bonnet extended Einstein equations', Nucl. Phys. B268, 737 (1986).

[8] R.C.Myers, J.Z. Simon, 'Black-hole thermodynamics in Lovelock gravity',Phys. Rev. D38, 2434 (1988).

[9] R. P. Woodard, 'The Theorem of Ostrogradsky' arXiv:1506.02210 [hepth].

[10] H. Motohashi and T. Suyama, 'Third order equations of motion and the Ostrogradsky instability', Phys. Rev. D 91, 085009 (2015). 
[11] C. Lanczos, 'A Remarkable property of the Riemann-Christoffel tensor in four dimensions', Annals Math39, 842 (1938).

[12] S. G. Ghosh and S. D. Maharaj, 'Cloud of strings for radiating black holes in Lovelock gravity', Phys. Rev. D 89, 084027 (2014); arXiv:1409.7874 [gr-qc].

[13] D. J. Gross and E. Witten, 'Superstring modifications of Einstein's equations', Nucl. Phys.B277, 1 (1986).

[14] D. J. Gross and J. H. Sloan, 'The quartic effective action for the heterotic string', Nucl. Phys.B291, 41 (1987).

[15] R.R. Metsaev and A. A. Tseytlin, 'Two-loop $\beta$-function for the generalized bosonic sigma model', Phys. Lett. B191, 354 (1987).

[16] B. Zwiebach, 'Curvature squared terms and string theories', Phys. Lett. B156, 315 (1985).

[17] R. R. Metsaevand A. A. Tseytlin, 'Order $\alpha$ (two-loop) equivalence of the string equations of motion and the $\sigma$-model Weyl invariance conditions: Dependence on the dilaton and the antisymmetric tensor ', Nucl. Phys.B293, 385 (1987).

[18] D. Glavan and C. Lin, 'Einstein-Gauss-Bonnet Gravity in 4D Spacetime', Phys. Rev. Lett. 124, 081301 (2020), arXiv:1905.03601 [gr-qc]].

[19] J. Arrechea, A. Delhom and A. J. Cano, 'Inconsistencies in fourdimensional Einstein-Gauss-Bonnet gravity', Chinese Phys. C 45013107 (2021), arXiv:2004.12998 [gr-qc].

[20] J. Arrechea, A. Delhom and A. J. Cano, 'Comment on Einstein-GaussBonnet Gravity in Four-Dimensional Spacetime',Phys. Rev. Lett. 125, 149002; arXiv:2009.10715 [gr-qc].

[21] M. Gurses, T. C. Sisman and B. Tekin, 'Comment on Einstein-GaussBonnet Gravity in Four-Dimensional Spacetime', Phys. Rev. Lett. 125, 149001 (2020).

[22] K. Aoki, M. A. Gorji and S. Mukohyama, 'A consistent theory of $D=4$ Einstein-Gauss-Bonnet gravity', Phys. Lett. B810, 135843 (2020); arXiv:2005.03859[gr-qc]]. 
[23] K. Aoki, M. A. Gorji and S. Mukohyama, 'Cosmology and gravitational waves in consistent $D \rightarrow 4$ Einstein-Gauss-Bonnet gravity', JCAP2020, 014 (2020); [ arXiv:2005.08428 [gr-qc]].

[24] H. Ghaffarnejad, E. Yaraie and M. Farsam, 'Quantum State Dependence of Thermodynamic Phase Transition in 4D AdS Gauss-Bonnet Quantum Black Holes Surrounded With Cloud of Strings', arXiv:2010.07108v5 [hep-th]

[25] T. H. Lee, D. Baboolal and S. G. Ghosh, 'Lovelock black holes in a string cloud background' EPJC 75, 297 (2015)

[26] J. M. Toledo and V. B. Beaerra, 'Black holes with cloud of strings and quintessence in Lovelock gravity' EPJC 78, 534 (2018)

[27] J. Maldacena, 'Black Holes and the Information Paradox in String Theory', www.ias.edu/ideas/2011/maldacena-black-holes-string-theory

[28] D. V. Singh, S. G. Ghosh and S. D. Maharaj, "Clouds of strings in $4 D$ Einstein-Gauss-Bonnet black holes",Phys.Dark Univ. 30 (2020) 100730 ; arXiv:2003.14136 [gr-qc]

[29] P. S. Letelier, 'Clouds Of Strings In General Relativity', Phys. Rev. D 20, 1294 (1979).

[30] B. Zwiebach, 'A first course in string theory', (Cambridge University press 2004).

[31] F. B. Hildebrand, 'Advanced Calculus for Application,', Prentice Hall, INC, (1962).

[32] L. Onsager, 'Statistical Hydrodynamics', Il, Nuovo Cim.6(Suppl2) (2): 279(1949).

[33] A. M. Tremblay, "Comment on Negative Kelvin temperatures: Some anomalies and a speculation", American Journal of Physics Vol. 44, No. 10, (1976) 


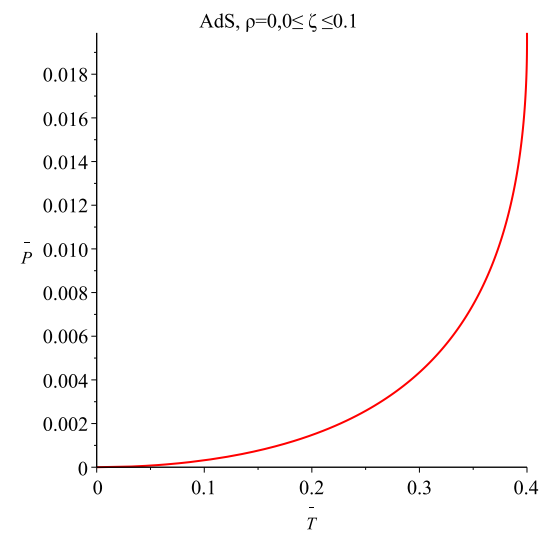

(a)

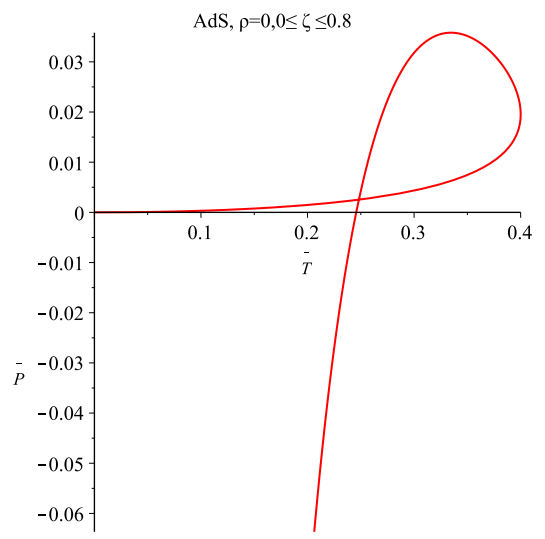

(c)

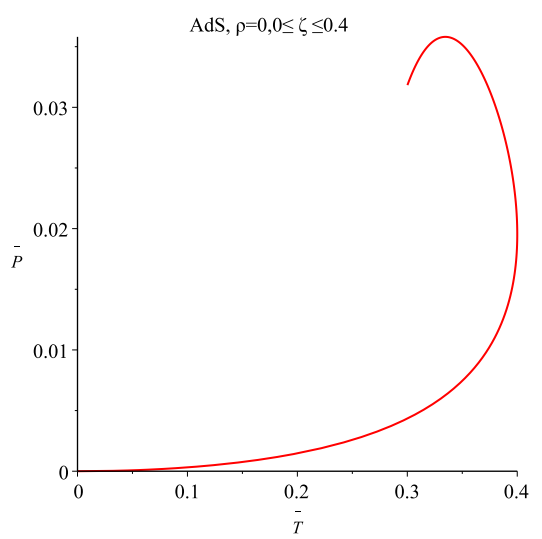

(b)

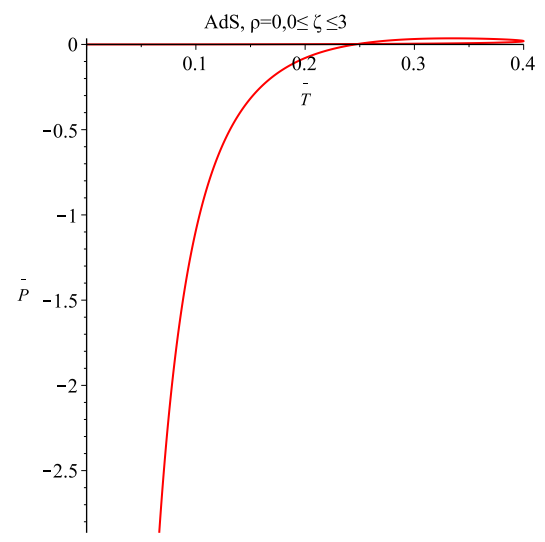

(d)

Figure 1: P-T diagrams for $\rho=0$ with AdS background. For dS sector diagrams are similar with these curves except where the pressures should be inverted as $\bar{P} \rightarrow-\bar{P}$ 


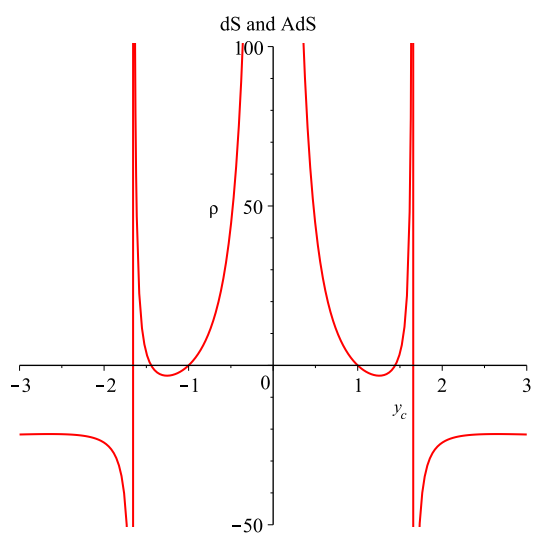

(a)

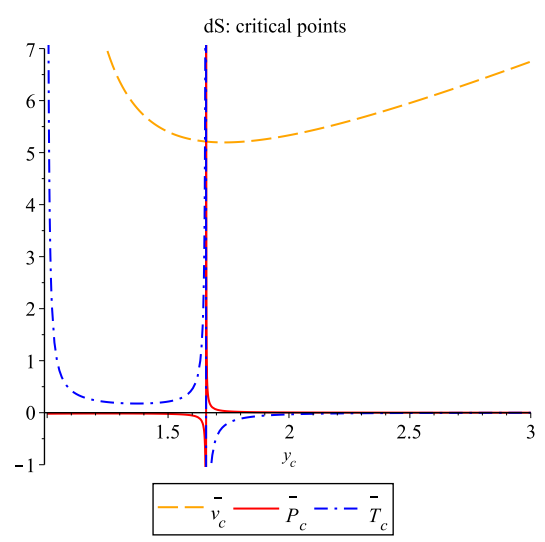

(b)

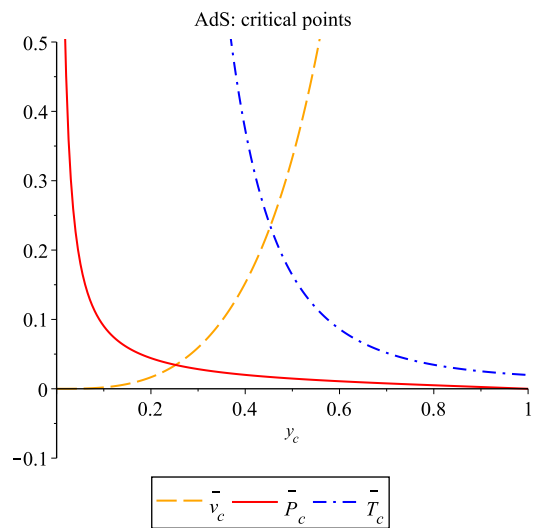

(c)

Figure 2: Numeric values of the critical points. 


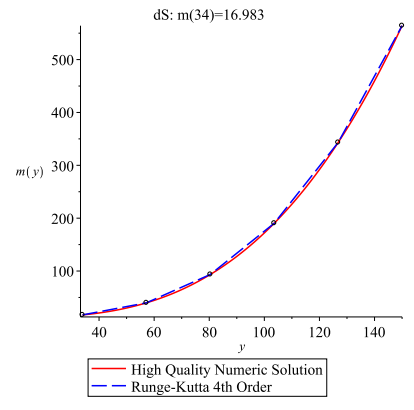

(a)

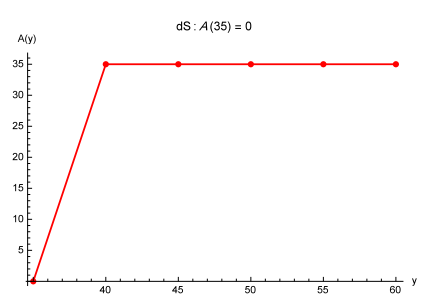

(b)

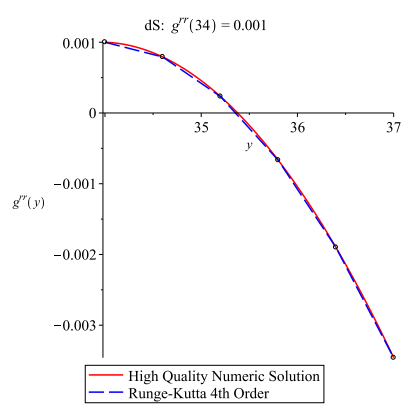

(c)

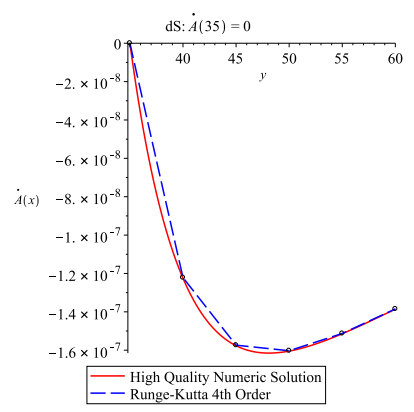

(d)

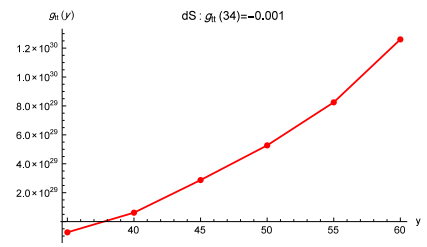

(e)

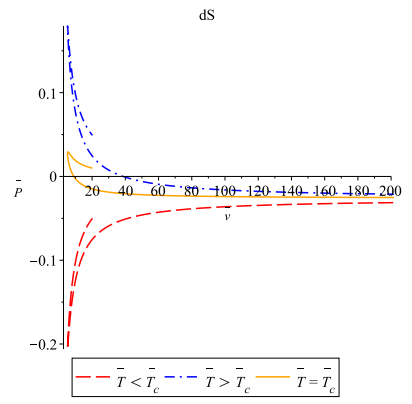

(f)

Figure 3: Diagrams of the numeric solutions of the fields with the $\mathrm{dS}$ background space. Initial values to produce numerical solutions are shown at top of each diagram. 


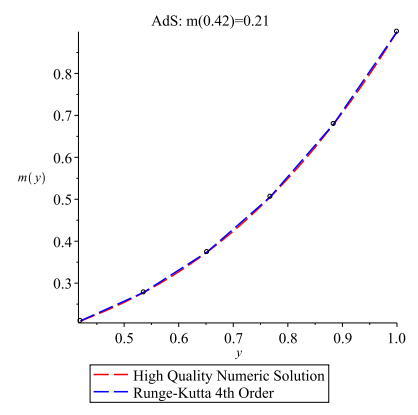

(a)

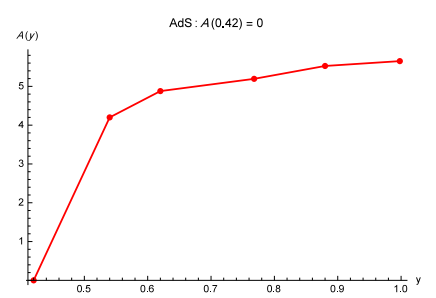

(b)

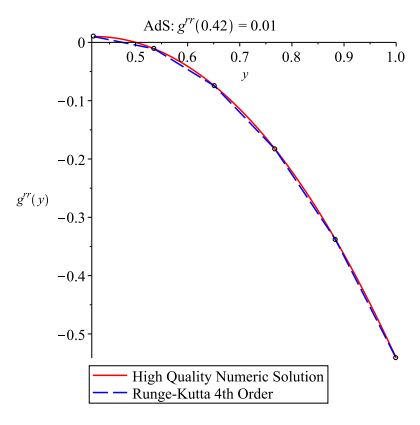

(c)

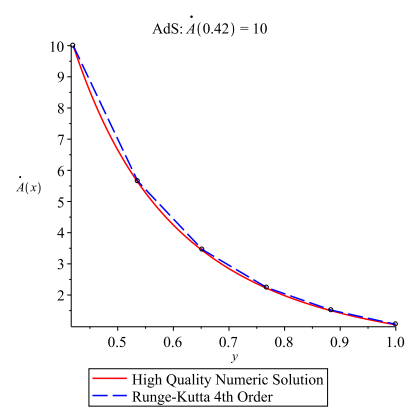

(d)

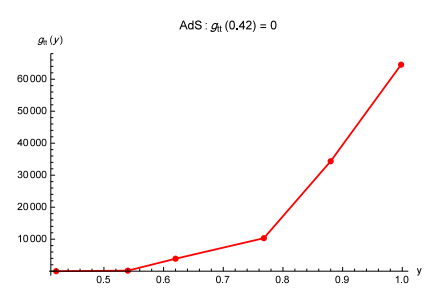

(e)

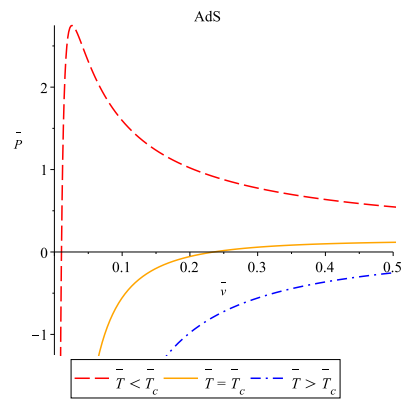

(f)

Figure 4: Numerical solutions are given by diagrams for AdS sector. Initial values to produce numerical solutions are shown at top of each diagram. 